\title{
Distribuição espacial e temporal da biomassa fitoplanctônica e variáveis ambientais no Golfão Maranhense, Brasil ${ }^{1}$
}

\author{
Andrea Christina Gomes de Azevedo ${ }^{2,4}$, Fernando A. Nascimento Feitosa ${ }^{3}$ e Maria Luise Koening ${ }^{3}$
}

Recebido em 24/01/2007. Aceito em 26/11/2007

\begin{abstract}
RESUMO - (Distribuição espacial e temporal da biomassa fitoplanctônica e variáveis ambientais no Golfão Maranhense, Brasil). Este trabalho foi desenvolvido com o objetivo de caracterizar alguns estuários do Golfão Maranhense, através da análise da biomassa fitoplanctônica e das variáveis ambientais. As amostras bimestrais foram coletadas em quatro pontos fixos, na superfície, em maré de sizígia, durante a vazante, no período de maio/2002 a março/2003. A pluviometria anual durante o período amostrado foi de 1.898,5 mm; a salinidade variou entre 6 e 35; transparência de 18 a $61 \mathrm{~cm}$; oxigênio dissolvido de 2,1 a $6,3 \mathrm{ml} . \mathrm{L}^{-1}$; taxa de saturação do oxigênio de 45 a $126 \%$; amônia de 0,02 a 26,45 $\mu \mathrm{M}$; nitrito de 0,01 a $0,25 \mu \mathrm{M}$; nitrato de 0,24 a 3,53 $\mu \mathrm{M}$ e fosfato de 0,13 a $0,58 \mu \mathrm{M}$. A concentração da clorofila $a$ variou de 1,92 a $72,23 \mathrm{mg} \cdot \mathrm{m}^{-3}$ caracterizando esta região como de alta produtividade e a fração < $20 \mu \mathrm{m}$ (nano e picofitoplâncton) foi considerada a mais representativa. A Análise dos Componentes Principais (ACP) evidenciou que a clorofila $a$ relacionou-se positivamente com a salinidade, transparência da água, velocidade do vento, fosfato e nitrato e inversamente com a pluviosidade, oxigênio dissolvido, taxa de saturação do oxigênio, temperatura e amônia. No Golfão Maranhense forçantes físicas como vento, macromaré e a alta turbidez são as principais causas da redução da zona eufótica e conseqüentemente da biomassa fitoplanctônica.
\end{abstract}

Palavras-chave: fitoplâncton, clorofila $a$, estuário, variáveis ambientais

\begin{abstract}
Spatial and temporal distribution of the phytoplanktonic biomass and environmental variables in the Maranhense Gulf, Brazil). Phytoplanktonic biomass and environmental parameters were investigated in order to characterize the estuaries of the Maranhense Gulf. The samples had been collected bimonthly at four fixed points, in the period of May/2002 to March/2003 in the surface, in ebb and spring tides. The annual pluviometry during the period was of $1.898,5 \mathrm{~mm}$; the salinity varied from 6 to 35 ; transparency from 18 to $61 \mathrm{~cm}$; dissolved oxygen from 2,1 to $6,3 \mathrm{ml} . \mathrm{L}^{-1}$; oxygen saturation from 45 to $126 \%$; ammonia from 0,02 to $26,45 \mu \mathrm{M}$; nitrite from 0,01 to $0,25 \mu \mathrm{M}$; nitrate from 0,24 to $3,53 \mu \mathrm{M}$ and phosphate of 0,13 to $0,58 \mu \mathrm{M}$. The total chlorophyll $a$ from 1,92 to $72,23 \mathrm{mg}$. $\mathrm{m}^{-3}$ and the fraction of nano and picophytoplankton from $0,86(15 \%)$ to $36,80 \mathrm{mg} \cdot \mathrm{m}^{-3}(97 \%)$, characterizing this area as quite productive. The principal component analysis (PCA) demonstrated that the chlorophyll $a$ had a direct relationship with the salinity, transparency of the water, speed of the winds, phosphate and nitrate and inverse correlation with the rain, dissolved oxygen, saturation of the oxygen, temperature and ammonia. In the Maranhense Gulf wind, macrotides and the high turbidity are the main causes in the reduction of the euphotic zone and consequently of the chlorophyll $a$.
\end{abstract}

Key words: phytoplankton, chlorophyll $a$, estuary, environmental variables

\section{Introdução}

Os sistemas estuarinos são os principais fornecedores de nutrientes para a região costeira, pois recebem e concentram o material originado de sua bacia de drenagem e podem vir a receber aportes significativos por ação antrópica. Todo esse aporte de nutrientes coloca os estuários entre os sistemas mais produtivos do mundo, com altas taxas de produtividade primária e teores de biomassa (Braga et al. 2000; Pereira Filho et al. 2001).

Estuários constituem ecótones entre os ambientes marinhos e de água doce, onde a salinidade muda constantemente. São sistemas ecológicos únicos, apresentando características fundamentais para o ciclo da vida de várias espécies de organismos; tornam-se áreas preferenciais de formação de núcleos urbanos por serem facilmente exploráveis e em razão dos recursos diversos que oferecem (Silva \& Koening 1993).

De acordo com Grego et al. (2004), as alterações ambientais nos estuários afetam, também, os organismos neles presentes, e dentre estes, encontra-se a comunidade fitoplanctônica, responsável pela base da teia trófica. Sob a ação de fatores bióticos e abióticos esta comunidade de algas apresenta variações sazonais e temporais em sua composição, biomassa e produtividade.

\footnotetext{
1 Parte da Tese de Doutorado da primeira Autora

2 Universidade Estadual do Maranhão, Departamento de Química e Biologia,Cidade Universitária Paulo VI s.n., Departamento de Química e Biologia, 65055-310 São Luis, MA, Brasil

3 Universidade Federal de Pernambuco, Programa de Pós-graduação em Oceanografia, 50670-901 Recife, PE, Brasil

4 Autora para correspondência: andreacgazevedo@uol.com.br
} 
O litoral maranhense é relativamente extenso e se destaca pela diversidade de feições geológicas, contendo de oeste para leste o Delta do Parnaíba, os Lençóis, o Golfão e as Reentrâncias Maranhenses. Além disso, possui uma ampla área de manguezais que representa mais de $40 \%$ das florestas de mangue do Brasil (Souza Filho 2005) o que aumenta consideravelmente a biodiversidade e a produtividade da zona costeira.

Apesar da complexidade de ecossistemas e de sua importância ecológica, os trabalhos desenvolvidos no Maranhão sobre biomassa fitoplanctônica ainda são muito escassos podendo-se citar o de Teixeira et al. (1988), analisando a produtividade e parâmetros hidrológicos no estreito dos Coqueiros; o de Alcântara et al. (2004) estudando a modelagem e a concentração de clorofila a no estuário do rio Anil; e Azevedo \& Cutrim (2007) considerando a distribuição do teor de biomassa total e fracionada do fitoplâncton no litoral norte-nordeste da ilha de São Luís. Portanto, com o intuito de ampliar os conhecimentos ecológicos no Golfão Maranhense este trabalho objetiva avaliar a distribuição espacial e temporal da biomassa fitoplanctônica e variáveis ambientais.

\section{Material e métodos}

O Estado do Maranhão, situado na porção norte brasileira contém litoral com extensão aproximada de $640 \mathrm{~km}$ e sua faixa litorânea possui características geoambienteais diferenciadas que justificam sua divisão em Litoral Ocidental, Golfão Maranhense e Litoral Oriental. A proximidade do equador e a configuração do relevo favorecem a amplitude das marés, alcançando 7,2 m (Feitosa \& Trovão 2006).

A ilha de São Luís possui área de aproximadamente $1.453 \mathrm{~km}^{2}\left(02^{\circ} 23^{\prime} 00^{\prime \prime}\right.$ - $02^{\circ} 47^{\prime} 00^{\prime \prime} \mathrm{S}$ e $44^{\circ} 00^{\prime} 29^{\prime \prime}$ $\left.44^{\circ} 24^{\prime} 29^{\prime \prime} \mathrm{W}\right)$ e está inserida na plataforma continental brasileira, no centro do Golfão Maranhense. São Luís separa a baía de São José a leste, com área de 77.500 km² da baía de São Marcos a oeste, com aproximadamente $23.600 \mathrm{~km}^{2}$ (Rios 2001).

O clima do município é do tipo quente e úmido, situando-se entre os padrões equatorial e tropical, com dois períodos estacionais bem distintos marcados pelas precipitações pluviométricas: um chuvoso (janeiro a junho) e outro de estiagem (julho a dezembro), com totais anuais superiores a $2.000 \mathrm{~mm}$ (Azevedo \& Cutrim 2007).

Os manguezais são abundantes e freqüentes na área, devido à grande quantidade de rios (Anil, Bacanga, dos Cachorros e Mearim na baía de São Marcos e Tibiri na baía de São José) formando numerosos estuários. A rede hidrográfica maranhense é formada por rios caudalosos, típicos de planície, caracterizada por baixo declive nos trechos médio e baixo.

Foram realizadas seis coletas bimestrais, em marés de sizígia durante a vazante, no período de maio/2002 a março/2003, em quatro pontos previamente definidos do Golfão Maranhense: ponto 1 - P1 (02 40 '49's e $44^{\circ} 21^{\prime} 47^{\prime}$ 'W) - próximo ao porto da Associação de Alumínio do Maranhão (ALUMAR); ponto 2 - P2 $\left(2^{\circ} 40^{\prime} 41^{\prime \prime} \mathrm{S}\right.$ e $\left.44^{\circ} 20^{\prime} 20^{\prime \prime} \mathrm{W}\right)$ na desembocadura do rio dos Cachorros; ponto 3 - P3 (2 $45^{\prime} 24^{\prime \prime} \mathrm{S}$ e $\left.44^{\circ} 21^{\prime} 43^{\prime \prime} \mathrm{W}\right)$, no estreito dos Mosquitos, todos na baía de São Marcos e ponto 4 - P4 (02 $44^{\prime} 10^{\prime \prime}$ S e $44^{\circ} 15^{\prime} 48^{\prime \prime} \mathrm{W}$ ) nas proximidades da desembocadura do rio Tibiri, baía de São José (Fig. 1).

Para a obtenção dos dados de clorofila $a$, as amostras foram coletadas na camada superficial da água com garrafa de Niskin, acondicionadas em frascos foscos e filtradas a vácuo. Os filtros utilizados foram os de fibra de vidro Whatman GF/C $(0,48 \mu \mathrm{m}$ de porosidade e $47 \mathrm{~mm}$ de diâmetro). Para cada ponto de coleta foram obtidas tréplicas utilizando volume de $250 \mathrm{ml}$. As amostras seguiram dois procedimentos, onde no primeiro houve filtração da clorofila $a$ total e no segundo a amostra foi passada por um copo com tela de $20 \mu \mathrm{m}$ para separar as diferentes frações da comunidade fitoplanctônica ( $>20 \mu \mathrm{m}$ microfitoplâncton e $<20 \mu \mathrm{m}$ nano e picofitoplâncton). $\mathrm{O}$ valor da clorofila $a$ para a fração do microfitoplâncton foi calculado pela diferença entre a clorofila $a$ total e o conteúdo de clorofila do nano e picofitoplâncton. Após a secagem, os filtros foram mantidos em freezer à temperatura de $-18{ }^{\circ} \mathrm{C}$ até a sua análise. A concentração de clorofila $a$ seguiu a metodologia usada para espectrofotometria conforme as recomendações descritas em Parsons \& Strickland

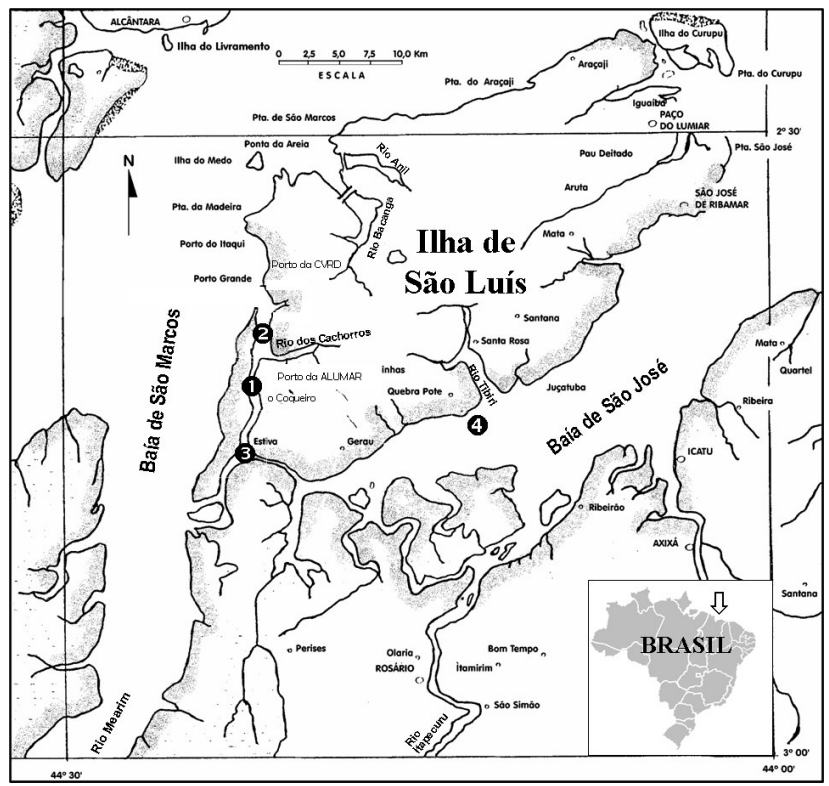

Figura 1. Localização dos pontos de coleta do fitoplâncton no Golfão 


\section{(1963) e UNESCO (1966).}

Foram aferidos os seguintes parâmetros: salinidade da água, pelo método de Winkler descrito por Strickland \& Parsons (1972)\%o; temperatura da água, com termômetro de mercúrio $\left(-10\right.$ a $\left.60{ }^{\circ} \mathrm{C}\right)$; transparência da água, com disco de Secchi; pH, com phmetro (HANNA); teor de oxigênio dissolvido (Strickland \& Parsons 1972), taxa de saturação (UNESCO 1973); sais nutrientes (nitrito, nitrato, amônia e fosfato) (Strickland \& Parsons 1972; Grasshoff et al. 1983). Os dados de pluviometria total, radiação solar diária, velocidade dos ventos e umidade relativa do ar procederam do Laboratório de Meteorologia da Universidade Estadual do Maranhão (LabMet/UEMA).

A Análise dos Componentes Principais (ACP) foi utilizada com a finalidade de evidenciar e hierarquizar os fatores responsáveis pela variância dos dados tendo sido aplicado o programa computacional NTSYS (Numerical Taxonomy and Multivariate Analysis System) da Metagrapics Software Corporation, Califórnia - USA.

\section{Resultados}

Os dados pluviométricos referentes ao período de abril/2002 a março/2003 registraram valor mínimo de $1,5 \mathrm{~mm}$ de chuva em setembro/2002 e máximo de $490 \mathrm{~mm}$ em abril/2002, tendendo a acompanhar a curva da média histórica de 28 anos (1970 a 1998). Foi possível constatar um ciclo sazonal definido, onde o período chuvoso foi de janeiro a junho, com máximas de precipitação em abril e o período de estiagem de julho a dezembro (Fig. 2).

Em relação ao total anual de chuvas, observou-se que durante o período de coleta a precipitação foi de $1.898,5 \mathrm{~mm} \cdot \mathrm{a}^{-1}$, próximo à média de onze anos (1.795,2 mm.a ${ }^{-1}, 1988$ a 1998).

A radiação média solar diária variou de $65,0 \mathrm{cal} . \mathrm{cm}^{-2} \cdot \mathrm{dia}^{-1} \mathrm{em}$ janeiro/2003 a 253,3 $\mathrm{cal} \cdot \mathrm{cm}^{-2} \cdot \mathrm{dia}^{-1}$

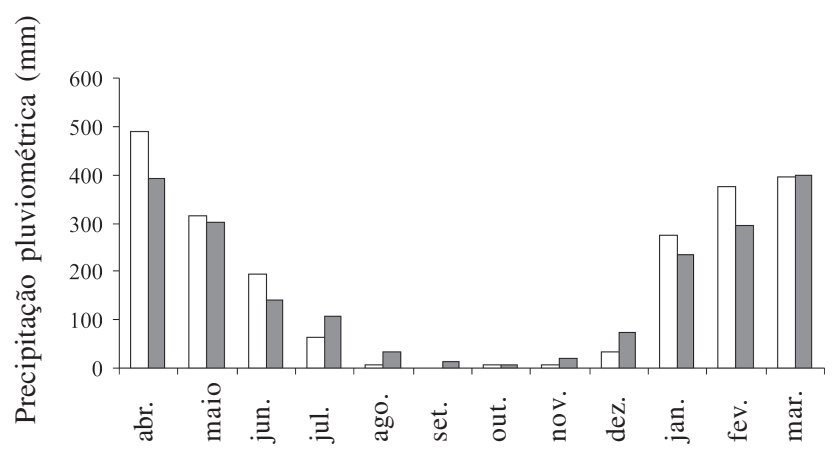

Figura 2. Total de chuvas (abril/2002 a março/2003) e média histórica mensal de chuvas (1970-1998). ( $\square$ = Total de chuvas; $\square$ = Média histórica). em maio/2002, ambos registrados no ponto 4. A velocidade dos ventos oscilou entre $2,0 \mathrm{~m} . \mathrm{s}^{-1}$ (janeiro/2003) e 11,6 m.s ${ }^{-1}$ (setembro/2002 e janeiro/2003) e a umidade relativa do ar registrou valores entre 72 e $88 \%$, em maio/2002 e janeiro/2003, respectivamente (Tab. 1).

Em relação à temperatura da água foi observada discreta variação sazonal, com amplitude de apenas $3{ }^{\circ} \mathrm{C}$. Os menores valores $\left(27^{\circ} \mathrm{C}\right)$ ocorreram em julho/2002, setembro/2002 e novembro/2002 e os maiores $\left(30^{\circ} \mathrm{C}\right)$ nos meses de julho/2002 e março/2003. Quanto à distribuição espacial verificou-se um gradiente decrescente de temperatura em direção ao ponto 4 , nos meses de maio/2002 e julho/2002, ocorrendo o processo inverso em setembro/2002, janeiro/2003 e março/2003 (Fig. 3).

Durante o período de estudo não foi evidenciada variação sazonal de $\mathrm{pH}$, e seus valores oscilaram entre 6,50 (ponto 4, maio/2002) e 8,03 (ponto 3 , julho/2002), mantendo-se alcalino (média 7,5). Foi possível constatar um gradiente decrescente de $\mathrm{pH}$ (neutro a ácido) em direção ao ponto 4 , ocorrendo o inverso apenas em março/2003 (Fig. 4).

O regime de salinidade nos estuários do Golfão Maranhense variou sazonalmente de meso a eualino, com amplitude total da salinidade de 29 e média de 21,3 . O valor mínimo foi de 6 (janeiro/2003 e março/2003, no ponto 3 e março/2003, no ponto 4) e máximo de 35 no ponto 1 (janeiro/2003). No ponto 3 foram registrados os menores valores de salinidade (Fig. 5).

Os valores de transparência da água estiveram mais baixos durante o período chuvoso (março/2003), variando de $18 \mathrm{~cm}($ março/2003, ponto 4$)$ a $61 \mathrm{~cm}(\mathrm{em}$ janeiro/2003, ponto 4). Os pontos 1 e 3 apresentaram os maiores e os menores valores de transparência da água, respectivamente (Fig. 6).

Os teores de oxigênio dissolvido foram maiores no mês de maio/2002 e menores em novembro/2002, variando de $2,1 \mathrm{ml} . \mathrm{L}^{-1}$, em janeiro/2003 (45\% de saturação do oxigênio) a 6,3 ml. $\mathrm{L}^{-1}$, em maio/2002 (126\% de saturação do oxigênio), caracterizando um ambiente isento de poluição orgânica, com exceção do ponto 3 (setembro/2002 e novembro/2002), que apresentou taxas de saturação de 45 e $48 \%$, respectivamente. Quanto à variação espacial os menores valores de oxigênio dissolvido e taxa de saturação do oxigênio foram registrados no ponto 3 e os maiores no ponto 4 , e sazonalmente foram menores durante o período de estiagem do que no período chuvoso (Fig. 7).

$\mathrm{O}$ teor de amônia foi baixo, exceto no ponto 2, em janeiro/2003 e em todos os pontos no mês de março/2003. As variações ocorreram de 0,02 (ponto 1 , janeiro/2003) a 26,45 $\mu \mathrm{M}$ (ponto 2, março/2003). A concentração de nitrito $(0,01$, ponto 1 e $0,25 \mu \mathrm{M}$, 
Tabela 1. Dados climatológicos registrados no dia da coleta, procedentes da estação meteorológica do LabMet/UEMA.

\begin{tabular}{|c|c|c|c|c|c|c|c|}
\hline Dados climatológicos & Pontos & Maio/2002 & Jul./2002 & Set./2002 & Nov./2002 & Jan./2003 & Mar./2003 \\
\hline \multirow[t]{2}{*}{ Radiação Solar $\left(\mathrm{cal} \mathrm{cm}^{-2} \cdot \mathrm{dia}^{-1}\right)$} & $\mathrm{P} 1 / \mathrm{P} 2 / \mathrm{P} 3$ & 192,8 & 192,8 & 345,0 & 240,4 & 149,3 & 243,0 \\
\hline & P4 & 253,3 & 209,7 & 217,0 & 159,0 & 65,0 & 183,9 \\
\hline \multirow[t]{2}{*}{ Velocidade dos Ventos $\left(\mathrm{m} \cdot \mathrm{s}^{-1}\right)$} & $\mathrm{P} 1 / \mathrm{P} 2 / \mathrm{P} 3$ & 7,9 & 9,1 & 8,8 & 11,0 & 11,6 & 6,5 \\
\hline & P4 & 9,9 & 8,0 & 11,6 & 10,7 & 2,0 & 4,6 \\
\hline \multirow[t]{2}{*}{ Umidade Relativa do $\operatorname{Ar}(\%)$} & $\mathrm{P} 1 / \mathrm{P} 2 / \mathrm{P} 3$ & 72,0 & 84,2 & 79,9 & 75,8 & 75,3 & 79,1 \\
\hline & $\mathrm{P} 4$ & 72,4 & 75,1 & 71,2 & 77,4 & 88,7 & 79,8 \\
\hline
\end{tabular}

ponto 3, novembro/2002) não apresentou padrão de sazonalidade (Fig. 8).

Foi possível constatar uma variação sazonal do nitrato no ponto 1 , com valores mais altos no período

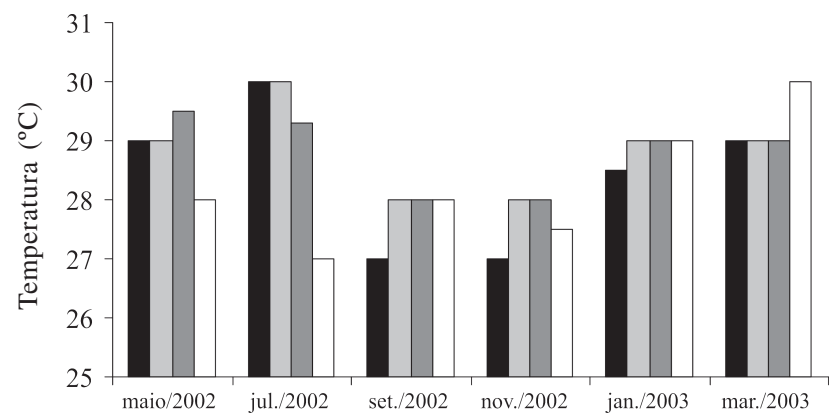

Figura 3. Variação espacial e temporal da temperatura da água nos estuários do Golfão Maranhense, Brasil. ( $\square=\mathrm{P} 1 ; \square=\mathrm{P} 2, \square=\mathrm{P} 3 ; \square=\mathrm{P} 4)$.

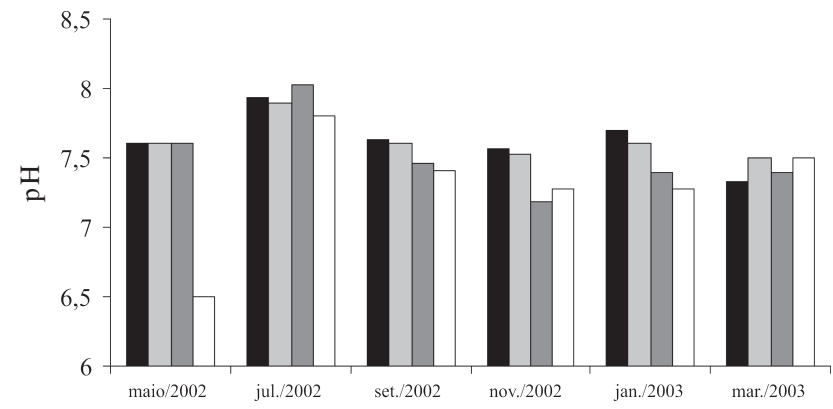

Figura 4. Variação espacial e temporal do potencial hidrogeniônico nos estuários do Golfão Maranhense, Brasil. ( $\square=\mathrm{P} 1 ; \square=\mathrm{P} 2, \square=\mathrm{P} 3$; $\square=\mathrm{P} 4)$.

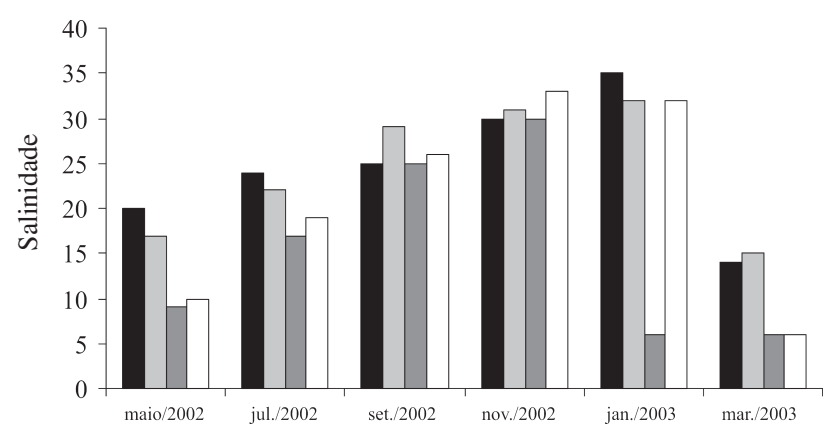

Figura 5. Variação espacial e temporal da salinidade da água nos estuários do Golfão Maranhense, Brasil. ( $\square=\mathrm{P} 1 ; \square=\mathrm{P} 2, \square=\mathrm{P} 3 ; \square=\mathrm{P} 4)$. chuvoso do que na estiagem, o oposto ocorrendo nos pontos 3 e 4, excetuando o mês de janeiro/2003. A variação foi de 0,24 (ponto 1) a $3,53 \mu \mathrm{M}$ (ponto 3), ambos em novembro/2002. O fosfato, entretanto, não mostrou um padrão sazonal; o ponto 3 registrou o menor valor e o ponto 4, o maior, com exceção do mês de março/2003. $\mathrm{O}$ teor de fosfato variou de 0,13 (março/2003, ponto 4) a $0,58 \mu \mathrm{M}$ (maio/2002, ponto 3) (Fig. 9).

Os valores de clorofila $a$ mostraram relação com os períodos estacionais, com a maior concentração no período de estiagem, especialmente nos meses de setembro/2002 e novembro/2002. A biomassa total variou de 1,92 (julho/2002, ponto 3 ) a $72,23 \mathrm{mg} \cdot \mathrm{m}^{-3}$ (novembro/2002, ponto 1), típico de ambiente eutrófico. O ponto 3 obteve os maiores valores em $66,7 \%$ das amostras analisadas.

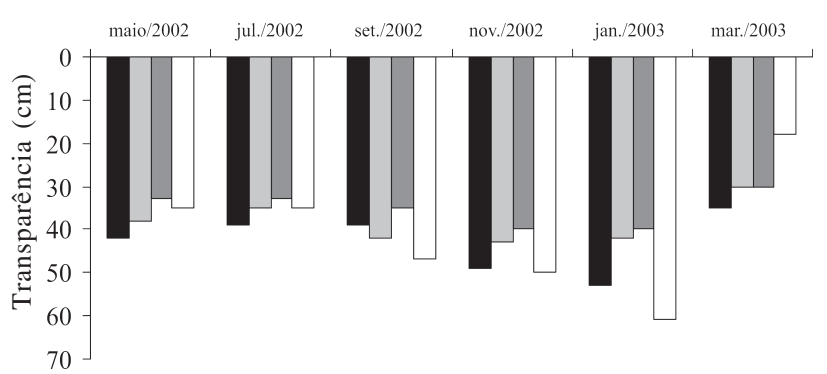

Figura 6. Variação espacial e temporal da transparência da água nos estuários do Golfão Maranhense, Brasil. ( $\square=\mathrm{P} 1 ; \square=\mathrm{P} 2, \square=\mathrm{P} 3$; $\square=$ P4).

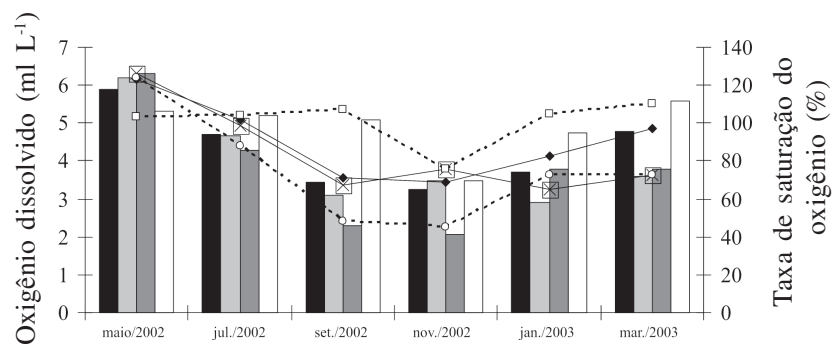

Figura 7. Variação espacial e temporal do oxigênio dissolvido (ml.L-1 $)$ em barras e taxa de saturação do oxigênio (\%) em linhas nos estuários do Golfão Maranhense, Brasil. ( $\square=\mathrm{P} 1 ; \square=\mathrm{P} 2, \square=\mathrm{P} 3 ; \square=\mathrm{P} 4$, $---=\mathrm{P} 1 ;-\times-=\mathrm{P} 2 ;--\mathrm{O}--=\mathrm{P} 3 ;--\square--=\mathrm{P} 4)$. 


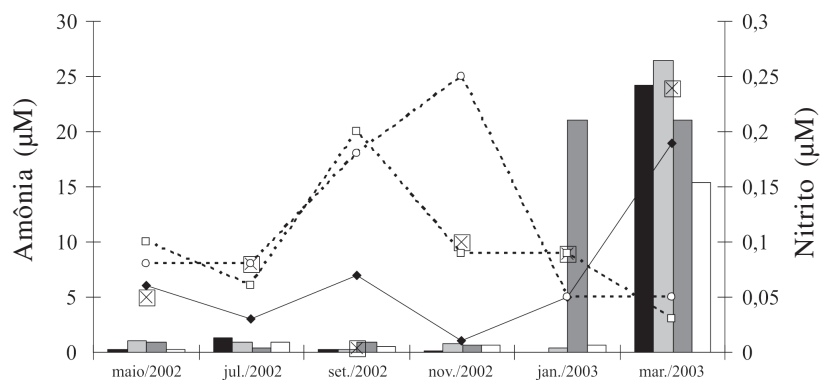

Figura 8. Variação espacial e temporal da amônia $(\mu \mathrm{M})$ em barras e nitrito $(\mu \mathrm{M})$ em linhas, nos estuários do Golfão Maranhense, Brasil. $(\square=\mathrm{P} 1 ; \square=\mathrm{P} 2, \square=\mathrm{P} 3 ; \square=\mathrm{P} 4,--=\mathrm{P} 1 ;-\times-=\mathrm{P} 2 ;--\mathrm{O}--=\mathrm{P} 3$; $--\square--=P 4)$.

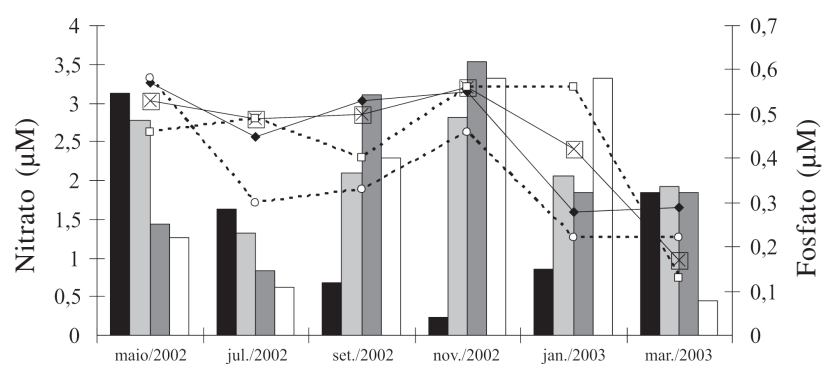

Figura 9. Variação espacial e temporal do nitrato $(\mu \mathrm{M})$ em barras e fosfato $(\mu \mathrm{M})$ em linhas, nos estuários do Golfão Maranhense, Brasil. $(\square=\mathrm{P} 1 ; \square=\mathrm{P} 2, \square=\mathrm{P} 3 ; \square=\mathrm{P} 4,-\bullet-=\mathrm{P} 1 ;-\times-=\mathrm{P} 2 ;--\mathrm{O}--=\mathrm{P} 3$; $--\square--=P 4)$.

Com relação aos valores de biomassa fracionada, identificou-se uma maior contribuição do nano/ picofitoplâncton, com valores superiores a $60 \%$ em $83 \%$ das amostras. A variação no fracionamento foi de 0,86 (15\%), em julho/2002 a 36,80 mg.m ${ }^{-3}(97 \%)$, em novembro/2002, ambos no ponto 4 , enquanto que o microfitoplâncton variou de $0,60 \mathrm{mg} \cdot \mathrm{m}^{-3}(31 \%)$, em julho/2002 a 36,80 mg.m ${ }^{-3}$ (90\%), em setembro/2002, ambos no ponto 3 (Fig. 10).

De acordo com a análise dos componentes principais (ACP) os três primeiros fatores explicaram $63,60 \%$ da variação dos dados: o fator 1 explicou 33,06\%, o fator 2 destacou $17,01 \%$ e o fator 3 explicou 13,53\%. (Tab. 2).

Analisando-se a projeção bidimensional dos primeiro e segundo fatores, do lado direito do observador, verifica-se que as variações de biomassa total e fracionada estão relacionadas com a salinidade, a transparência da água, o fosfato, a velocidade dos ventos e o nitrato, em ordem decrescente de importância no ambiente estuarino do Golfão Maranhense, relacionando-os ao déficit hídrico, com maiores valores de clorofila $a$ no período de estiagem. Do lado esquerdo do observador identificam-se os mais elevados índices pluviométricos correlacionados diretamente com a taxa de saturação do oxigênio, amônia, temperatura e oxigênio dissolvido na água (Fig. 11).

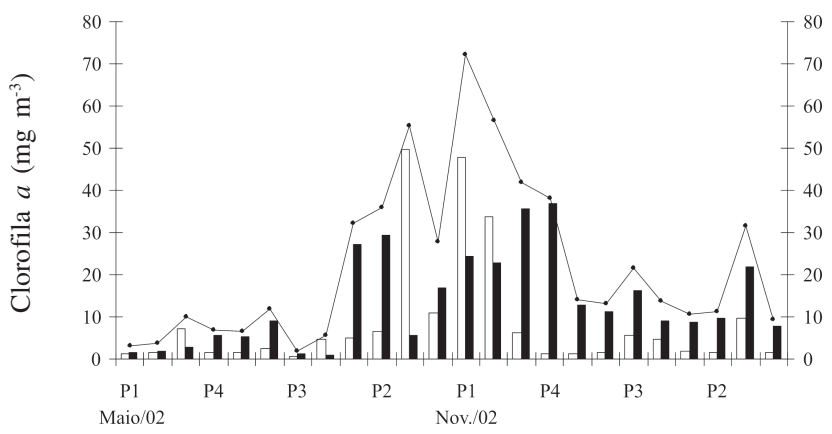

Figura 10. Variação espacial e temporal do microfitoplâncton, nano e picofitoplâncton $\left(\mathrm{mg} \cdot \mathrm{m}^{-3}\right)$, em barras e biomassa total $\left(\mathrm{mg} \cdot \mathrm{m}^{-3}\right) \mathrm{em}$ linha nos estuários do Golfão Maranhense. ( $\square=$ Microfitoplâncton; Ш = Nano e picofitoplâncton, $---=$ Biomassa total).

Tabela 2. Análise dos componentes principais (ACP) da biomassa fitoplanctônica e variáveis ambientais no Golfão Maranhense, Brasil.

\begin{tabular}{|c|c|c|c|c|}
\hline Variáveis Ambientais & Abreviações na ACP & $\begin{array}{c}\mathrm{F} 1 \\
33,06 \%\end{array}$ & $\begin{array}{c}\mathrm{F} 2 \\
17,01 \%\end{array}$ & $\begin{array}{c}\text { F3 } \\
13,53 \%\end{array}$ \\
\hline Biomassa total & BT & 0,6501 & 0,5190 & 0,0315 \\
\hline Biomassa fracionada (nano e picofitoplâncton) & $\mathrm{BF}$ & 0,6222 & 0,5016 & 0,1674 \\
\hline Altura da maré & Maré & 0,3230 & $-0,2137$ & $-0,7709$ \\
\hline Temperatura da água & Temp & $-0,6245$ & $-0,4320$ & 0,3102 \\
\hline Salinidade & Sal & 0,9057 & $-0,2090$ & 0,2089 \\
\hline Pluviometria & Pluv & $-0,8542$ & 0,0069 & $-0,0727$ \\
\hline Radiação solar acumulada & Rad.Sol. & $-0,0901$ & 0,6925 & $-0,4346$ \\
\hline Transparência da água & Secchi & 0,7424 & $-0,2997$ & 0,1961 \\
\hline Velocidade do vento & Vento & $\mathbf{0 , 5 5 7 1}$ & 0,2826 & $-0,3292$ \\
\hline Umidade relativa do ar & Umid & 0,0516 & $-0,4134$ & 0,5804 \\
\hline Oxigênio dissolvido & $\mathrm{OD}$ & $-0,6034$ & $-0,5098$ & $-0,4386$ \\
\hline Taxa de saturação do oxigênio & Txsat & $-0,7933$ & 0,3609 & $-0,3287$ \\
\hline pH da água & $\mathrm{pH}$ & 0,0112 & $-0,5093$ & $\mathbf{0 , 0 5 8 6}$ \\
\hline Concentração de amônia & Amônia & $-0,6657$ & 0,4754 & 0,4319 \\
\hline Concentração de nitrito & Nitrito & 0,0311 & 0,4897 & 0,2410 \\
\hline Concentração de nitrato & Nitrato & 0,3312 & 0,0391 & 0,2496 \\
\hline Concentração de fosfato & Fosfato & 0,5886 & $-0,3968$ & $-0,4980$ \\
\hline
\end{tabular}




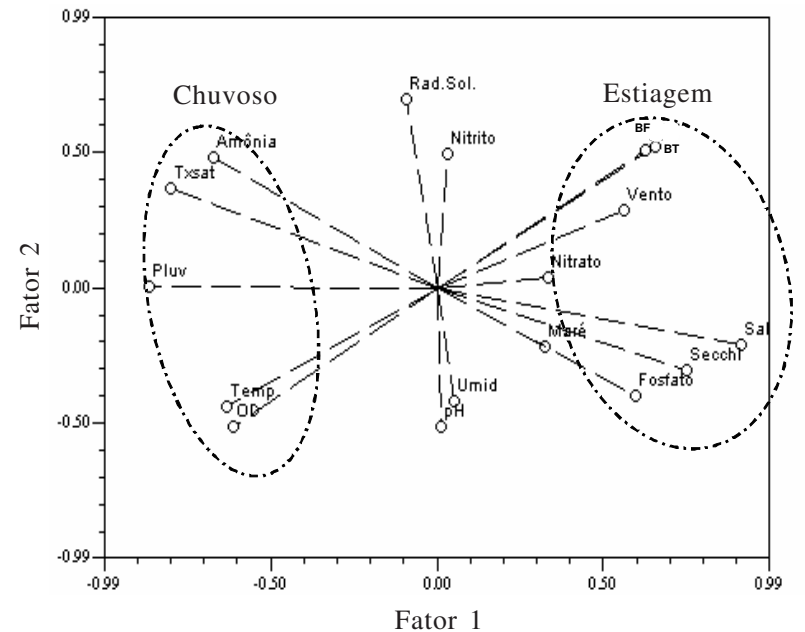

Figura 11. Contribuição da biomassa fitoplanctônica e parâmetros ambientais referentes aos dois primeiros componentes principais, nos estuários do Golfão Maranhense, Brasil.

\section{Discussão}

Na região do Golfão Maranhense a pluviometria esteve dentro do padrão esperado, condicionando alterações sazonais nos parâmetros oceanográficos. Foi verificada uma correlação direta entre a biomassa fitoplanctônica, a salinidade, a transparência da água, o fosfato, a velocidade dos ventos e o nitrato e relação inversa da pluviometria com a taxa de saturação do oxigênio, amônia, temperatura e oxigênio dissolvido na água. No entanto, a radiação solar, o nitrito, $\mathrm{pH}$, umidade relativa do ar e as marés, não interferiram nas variações do teor de biomassa, confirmados pela Análise dos Componentes Principais (ACP).

Em ambientes estuarinos, as variações ambientais estão relacionadas com fatores climatológicos como precipitação pluviométrica, radiação solar e movimentos sazonais da água, que são a circulação local e influência de correntes costeiras pela ação dos ventos (Tundisi 1970).

A determinação da biomassa fitoplanctônica através da extração da clorofila $a$ é um dos métodos mais precisos e dela é possível avaliar a comunidade dos produtores primários aquáticos. Os teores de biomassa total e fracionada foram maiores no período de estiagem, da mesma forma como na Bacia do Pina (Feitosa \& Passavante 1990), no estuário do rio Goiana, PE (Feitosa et al. 1999) e no estuário dos rios Ilhetas e Mamucaba, em Tamandaré, PE (Losada et al. 2003). Ao contrário, nos estuários do rio Una (Bastos et al. 2005) e do rio Jaguaribe, PE (Santos-Fernandes et al. 1998), as maiores taxas de clorofila $a$ foram observadas durante o período chuvoso.

O teor de clorofila $a$ nos estuários do Golfão Maranhense indica que esta região é bastante produtiva, alcançando valores de 72,3 mg.m $\mathrm{m}^{-3}$, superando outras áreas estuarinas, como os estuários do rio Una (1,68 a $36,30 \mathrm{mg} \cdot \mathrm{m}^{-3}$ ) (Bastos et al. 2005), o estuário do rios Jaguaribe (1,35 a 38,47 mg.m $\mathrm{m}^{-3}$ ) (Santos-Fernandes et al. 1998) e o estuário de Barra das Jangadas $(0,57$ a 59,84 mg.m ${ }^{-3}$ ) (Branco et al. 2002).

Teixeira et al. (1988) ao realizarem coletas pontuais no estreito dos Coqueiros - MA, identificaram que a camada eufótica era extremamente reduzida, sendo quase toda a produção primária realizada na camada superficial $(<0,50 \mathrm{~m})$. Tal observação redunda num ecossistema caracterizado por um grande potencial fotossintético, porém restrito a uma camada delgada de água em decorrência da alta turbidez.

Em outras regiões similares a coluna d'água iluminada alcança 4,30 m (Teixeira et al. 1969) e 4,70 m (Losada et al. 2003), sugerindo que, independentemente da origem, o material particulado deve ter papel muito importante dentro da teia trófica deste ecossistema, embora seja o principal causador da turbidez das águas.

A variabilidade espacial e temporal da dinâmica fitoplanctônica pode ser explicada pela variação da razão entre as zonas afótica/zona eufótica como observado no estuário de Gironde, sudeste da França, onde a produção primária pode ser limitada pela luz e dependente das condições hidrodinâmicas (Irigoien \& Castel 1997).

Tem-se registrado que a fração do nanoplâncton é responsável por 80 a $100 \%$ da produtividade e clorofila (Santos-Fernandes et al. 1998). Este padrão também foi observado ao comparar os resultados com dados pretéritos reportados por Teixeira et al. (1988) no estreito dos Coqueiros, MA, ao observarem que a fração do nanofitoplâncton correspondeu a $86 \%$ da biomassa e no atual trabalho a fração $<20 \mu \mathrm{m}$ correspondeu até $97 \%$ da clorofila. A luz é considerada como um dos fatores limitantes para a produção primária. Como a transparência da água varia em função da chuva e da drenagem terrestre, pode-se esperar que a luz seja fator limitante para o fitoplâncton nos meses de maior pluviometria, podendo limitar a produção potencial da biomassa fitoplanctônica (Passavante \& Koening 1984; Sassi 1991). O mesmo foi constatado nos estuários do Golfão Maranhense, onde o período de maior índice pluviométrico coincidiu com o menor teor de clorofila $a$ e a menor transparência na água, portanto, dificultando a assimilação dos nutrientes disponibilizados pelo carreamento das chuvas, por diminuição da camada eufótica.

A turbidez e ação das macromarés ( $>6 \mathrm{~m}$ ) são as principais causas da pequena espessura da zona eufótica devido à rápida atenuação da energia luminosa, causando assim redução da absorção da luz pelo fitoplâncton. Tais variáveis no corpo hídrico, além de causar acentuada compressão da camada iluminada, propiciam também a 
ocorrência de alta frequiência da flutuação da luz (Teixeira et al. 1988).

A ressuspensão dos sedimentos de fundo é causada muitas vezes pelos ventos. Segundo Pedersen et al. (1995), a velocidade mínima necessária para criar o envolvimento do fundo é estimada em $3 \mathrm{~m} \cdot \mathrm{s}^{-1}$. No estuário do rio Sem, na Alemanha, a velocidade média de $6 \mathrm{~m} . \mathrm{s}^{-1}$ foi considerada importante fator nos processos de ressuspensão (Jonge \& Beusekom 1995). A velocidade do vento atingiu $11,6 \mathrm{~m} \cdot \mathrm{s}^{-1}$, com média de $8,5 \mathrm{~m} \cdot \mathrm{s}^{-1} \mathrm{em}$ torno da ilha de São Luís - MA, levando-se a crer que este parâmetro físico interferiu na baixa transparência da água. A temperatura é uma variável física que assume grande importância na produtividade biológica da água, cujos ritmos dependem dela, sendo considerada um dos fatores ecológicos decisivos dentro dos sistemas e o principal limitante da distribuição geográfica de muitas espécies de vegetais e animais (Sipaúba-Tavares 1998). Nos estuários tropicais a temperatura é sempre elevada e as pequenas variações que ocorrem durante o ciclo sazonal, dependem do grau de insolação e de outras condições meteorológicas (Honorato da Silva et al. 2004). No Golfão Maranhense a temperatura da água mostrou discretos padrões de sazonalidade, sendo registrada amplitude de apenas $3^{\circ} \mathrm{C}$, como era de se esperar em áreas de tão baixas latitudes, e correlacionou-se negativamente com a biomassa fitoplanctônica.

Um dos parâmetros fundamentais para o desenvolvimento de uma comunidade em estuários é a salinidade, tornando-se fator condicionante para o estabelecimento das espécies, visto que pode oscilar em função da maré e da vazão dos rios, e os organismos que habitam estas áreas precisam estar adaptados às periódicas mudanças salinas. Além de servir tanto para delimitar o início e o término de um estuário, como também, influenciar a distribuição dos organismos, podendo ser considerada como uma barreira ecológica para as espécies conhecidas como estenoalinas (Feitosa et al. 1999). A salinidade mostrou-se nitidamente variável sazonalmente no Golfão Maranhense, com amplitude de 29 e média de 21,3 e maiores teores durante o período de estiagem, concordando com trabalhos de biomassa fitoplanctônica desenvolvidos em áreas estuarinas, dentre outros: Santos et al. (2000) no estuário do rio Jaguaribe, PE; Honorato da Silva et al. (2004) no estuário do rio Formoso, PE, Grego et al. (2004) no estuário do rio Timbó, PE.

Figueiredo et al. (2006) ao estudarem a variação dos parâmetros hidrológicos no Canal de Santa Cruz, PE observaram que a salinidade foi estável na estiagem, devido ao pequeno fluxo de água doce, à evaporação e maior incidência da radiação solar, enquanto que no período chuvoso, com a influência da descarga fluvial, houve uma pequena estratificação salina. Os menores valores de salinidade encontraram-se no ponto 3, próximo à desembocadura do rio Mearim, por estar mais distante do input salino advindo das baías de São Marcos e São José.

Nos estuários, as concentrações de oxigênio variam muito em função do fluxo e refluxo das marés. A importância da sua determinação se deve ao fato dele ser um gás essencial à vida e um indicador das condições ambientais (Bastos et al. 2005). Neste trabalho, o teor de oxigênio dissolvido e a taxa de saturação do oxigênio foram menores durante o período de estiagem e mais elevados no período chuvoso. Em relação à distribuição temporal, foram menores no ponto 3 (maior influência fluvial) e maiores no ponto 4 (menor influência fluvial), fato este, relacionado às variações de salinidade e temperatura durante o período de estudo.

$\mathrm{O}$ pH no Golfão Maranhense apresentou-se ligeiramente alcalino $(6,50$ a 8,03$)$, encontrando-se dentro dos limites aceitáveis para a vida marinha, conforme Perkins (1977) que comenta tais variações entre 6,5 e 9,0. Padrão semelhante foi encontrado por Branco et al. (2002) em Barra das Jangadas, PE, Honorato da Silva et al. (2004) no estuário do rio Formoso - PE e Bastos et al. (2005) que estudaram a biomassa fitoplanctônica no estuário do rio Una, PE.

Os principais elementos nutrientes que podem limitar a produção de matéria orgânica pelo fitoplâncton nos ecossistemas aquáticos são o nitrogênio, nas várias formas inorgânicas, e o fósforo (Tundisi \& Tundisi 1976). O nitrito e o fosfato podem limitar a produção orgânica, considerando que a luz não seja um dos fatores limitantes (Heip et al. 1995). As maiores concentrações de nitrito e nitrato nos estuários do Golfão Maranhense tenderam a ocorrer durante o período de estiagem, correspondendo aos maiores registros de biomassa.

Campelo et al. (1999) na praia de Carne de Vaca, PE detectaram que quanto maior o valor do fosfato, mais elevado foi o teor clorofila $a$ e Cavalcanti et al. (1991) no Canal de Santa Cruz, PE observaram relação direta entre os teores de nitrato e de biomassa, fatos constatados também neste trabalho.

De um modo geral, as concentrações médias de nitrito e fosfato, obtidas ao longo do período estudado estiveram dentro da faixa dos limites encontrados em áreas estuarinas do rio Jaguaribe, PE e no estuário do rio Una, $\mathrm{PE}$, enquanto que o nitrato apresentou valores inferiores (Santos et al. 2000; Bastos et al. 2005).

Fato importante foi o alto teor de amônia observado em janeiro/2003 no ponto 3 e em março/2003 nos quatro pontos de amostragem, tendo em vista que este nutriente registrou valores baixos na maioria dos meses amos- 
trados. Vale ressaltar que a elevação deste nutriente indica forte aporte de matéria orgânica no ecossistema analisado.

No Golfão Maranhense forçantes físicas como vento, macromarés e a alta turbidez são as principais causas da redução da zona eufótica e conseqüentemente da biomassa fitoplanctônica.

\section{Referências bibliográficas}

Alcântara, E.H.; Rebelo-Mochel, F.; Amorim, A.J.E. \& Thevand, A. 2004 Modelagem da profundidade secchi e da concentração de clorofila $a$ no estuário do rio Anil, São Luís - MA. Caminhos da Geografia 2: 19-40.

Azevedo, A.C.G. \& Cutrim, M.V.J. 2007. Fitoplâncton costeiro das porções norte-nordeste da ilha de São Luís, MA, Brasil. Pp. 67-92. In: A.C. Silva \& J.L.O. Fortes (orgs.). Diversidade biológica, uso e conservação de recursos naturais do Maranhão. São Luís, Universidade Estadual do Maranhão.

Bastos, R.B.; Feitosa, F.A.N. \& Muniz, K. 2005. Variabilidade espaço-temporal da biomassa fitoplanctônica e hidrológica no estuário do rio Una (PernambucoBrasil). Tropical Oceanography 33: 1-18.

Braga, E.S.; Bonetti, C.V.D.H.; Burone, L. \& Bonetti Filho, J. 2000. Eutrophication and bacterial pollution caused by industrial and domestic wastes at the Baixada Santista estuarine system - Brazil. Marine Pollution Bulletin 40: 165-173.

Branco, E.S.; Feitosa, F.A.N. \& Flores-Montes, M.J. 2002. Variação sazonal e espacial da biomassa fitoplanctônica relacionada com parâmetros hidrológicos no estuário de Barra das Jangadas (Jaboatão dos Guararapes Pernambuco - Brasil). Tropical Oceanography 30: 79-96.

Campelo, M.J.A.; Passavante, J.Z. \& Koening, M.L. 1999. Biomassa fitoplanctônica (clorofila $a$ ) e parâmetros ambientais na praia de Carne de Vaca, Goiana, Pernambuco, Brasil. Trabalhos Oceanográficos da Universidade Federal de Pernambuco 27: 27-41.

Cavalcanti, L.B; Macêdo, S.J. \& Passavante, J.Z. 1991. Estudo ecológico da região de Itamaracá, Pernambuco - Brasil. XXI - Caracterização do Canal de Santa Cruz em função dos parâmetros físico-químicos e pigmentos fotossintéticos. Trabalhos Oceanográficos da Universidade Federal de Pernambuco 16: 157-226.

Corson, W. H. 2002. Manual global de ecologia: o que você pode fazer a respeito da crise do meio ambiente. São Paulo, Augustus.

Feitosa, A.C. \& Trovão, J.R. 2006. Atlas escolar do Maranhão: espaço geohistórico e cultural. João Pessoa, Grafset.

Feitosa, F.A.N. \& Passavante, J.Z.O. 1990. Variação sazonal da produção primária na bacia do Pina (Recife - PE). Trabalhos Oceanográficos da Universidade Federal de Pernambuco 21: 33-46.

Feitosa, F.A.N.; Silva-Cunha, M.G.G; Passavante, J.Z.O.; Neumann-Leitão, S. \& Lins, I.A. 1999. Estrutura do microfitoplâncton no sistema estuarino do rio Goiana, Pernambuco, Brasil. Trabalhos Oceanográficos da Universidade Federal de Pernambuco 27: 15-25.

Figueiredo, J.A.; Muniz, K.; Macêdo, S.J.; Flores-Montes, M.J. \& Feitosa, F.A.N. 2006. Hidrologia e biomassa fitoplanctônica nas barras Orange e Catuama (Canal de Santa Cruz), em Itamaracá - PE: variação nictemeral. Arquivo de Ciências do Mar 39: 5-17.

Grasshof, K.; Ehrhardt, M. \& Kremling, K. 1983. Methods of seawater analysis. $2^{\text {nd }} \mathrm{ed}$. New York, Verlag Chemie.

Grego, C.K.S.; Feitosa, F.A.N.; Honorato da Silva, M. \& Flores-Montes, M.J. 2004. Distribuição espacial e sazonal da clorofila $a$ fitoplanctônica e hidrologia do estuário do rio Timbó (Paulista - PE). Tropical Oceanography 32: 181-199.

Heip, C.R.H.; Goosen, N.K.; Herman, P.M.J.; Kromkamp, J.; Middelburg, J.J. \& Soetaert, K. 1995. Production and consumption of biological particles in temperate tidal estuaries. Oceanography and Marine Biology: An Annual Review 33: 1-150.

Honorato da Silva, M; Passavante, J.Z.O.; Silva-Cunha, M.G.G.; Nascimento Vieira, D.A.; Grego, C.K.S. \& Muniz, K. 2004. Distribuição espacial e sazonal da biomassa fitoplanctônica e dos parâmetros hidrológicos do estuário do rio Formoso (Rio Formoso, Pernambuco, Brasil). Tropical Oceanography 32: $89-106$
Irigoien, X. \& Castel, J. 1997. Light limitation and distribution of chlorophyll pigments in a highly turbid estuary: the Gironde (SW France). Estuarine, Coastal and Shelf Science 44: 207-517.

Jonge, V.N. \& Beusekom, J.E.E. 1995. Wind and tide induced resuspension of sediment and microphytobenthos from tidal flats in the Ems estuary. Limnology and Oceanography 40: 766-778.

Losada, A.P.M.; Feitosa, F.A.N. \& Lins, I.C. 2003. Variação sazonal e espacial da biomassa fitoplanctônica nos estuários dos rios Ilhetas e Mamucaba (Tamandaré - PE) relacionada com parâmetros hidrológicos. Tropical Oceanography 31: 1-29.

Parsons, T.R. \& Strickland, J.D.H. 1963. Discussion of spectrophotometric determination of marine plankton pigments, with revised equations of as certaining chlorophyll $a$ and carotenoids. Journal of Marine Research 21: $155-163$.

Passavante, J.Z.O. \& Koening, M.L. 1984. Estudo ecológico da região de Itamaracá (Pernambuco - Brasil). XXVI Clorofila $a$ e material em suspensão no estuário do rio Botafogo. Trabalhos Oceanográficos da Universidade Federal de Pernambuco 18: 207-230.

Pedersen, O.B.; Christiansen, C. \& Laursen, M.B. 1995. Wind induced long term increase and short term flutuations of shallow waters suspend mater and nutrient concentrations, Ringkobing Fjord, Denmark. International Journal of Marine Biology 41: 273-287.

Pereira Filho, J.; Schettini, C.A.F.; Rörig, L. \& Siegle, E. 2001. Intratidal variation and net transport of dissolved inorganic nutrients, POC and chlorophyll $a$ in the Camboriu river estuary, Brazil. Estuarine, Coastal and Shelf Science 53: 249-257.

Perkins, E.J. 1977. Inorganic Wastes. Pp. 70-101. In: J. Lenihan \& W.W. Fletcher (eds). The Marine Environment. Glasgow, Blackie \& Son Limited.

Rios, L. 2001. Estudos de Geografia do Maranhão. $3^{\text {a }}$ ed. São Luís, Graphis.

Santos, T.L.; Passavante, J.Z.O.; Koening, M.L.; Macêdo, S.J. \& Lins, I.C. 2000. Fitoplâncton do estuário do rio Jaguaribe (Itamaracá, Pernambuco, Brasil): produção e hidrologia. Revista Ecológica Aquática Tropical 10: 43-69.

Santos-Fernandes, T.L.; Passavante, J.Z.O.; Koening, M.L. \& Macêdo, S. J. 1998. Fitoplâncton do estuário do rio Jaguaribe (Itamaracá, Pernambuco, Brasil): Biomassa. Trabalhos Oceanográficos da Universidade Federal de Pernambuco 26: 1-18.

Sassi, R. 1991. Phytoplankton and environmental factors in the Paraíba do Norte river estuary, southeastern Brazil: composition, distribution and quantitative remarks. Boletim do Instituto Oceanográfico 39: 93-115.

Silva, I.G. \& Koening, M.L. 1993. Variação sazonal da densidade fitoplanctônica no estuário do rio Paripe, Itamaracá - Pernambuco, Brasil. Arquivos de Biologia e Tecnologia 36: 645-658.

Sipaúba-Tavares, L.H. 1998. Limnologia dos sistemas de cultivos. Pp. 47-75. In: W.C. Valentin (ed.). Carcinicultura de Água Doce: Tecnologia para produção de camarões. Brasília, IBAMA/FAPESP.

Souza Filho, P.W.M. 2005. Costa de manguezais de macromaré da Amazônia: cenários morfológicos, mapeamento e quantificação de áreas usando dados de sensores remotos. Revista Brasileira de Geofísica 23: 427-435.

Strickland, J.D. \& Parsons, T.S. 1972. A pratical handbook of sea water analysis. $2^{\text {nd }}$ ed. Bulletin Fisheries Research Board of Canadá 167: 1-205.

Teixeira, C.; Tundisi, J. \& Santoro, J.Y. 1969. Plankton studies in a mangrove environment. VI. Primary production, zooplankton standing-stock and some environmental factors. Internationale Revue der Gesamten Hydrobiologie 54: 289-301.

Teixeira, C.; Aranha, F.J.; Barbieri, R. \& Melo, O.T. 1988. Produção primária e clorofila $a$ do fitoplâncton e parâmetros físicos e químicos do estreito dos Coqueiros - Maranhão - Brasil. Revista Brasileira de Biologia 48: 29-39.

Tundisi, I.G. 1970. O plâncton estuarino. Contribuições avulsas do Instituto Oceanográfico da Universidade de São Paulo, Série Oceanografia Biológica: 1-12.

Tundisi, J. \& Tundisi, T.M. 1976. Produção orgânica em ecossistemas aquáticos. Ciência e Cultura 28: 861-867.

UNESCO. 1966. Determination of photosyntetic pigments in sea water. Paris, UNESCO.

UNESCO. 1973. International Oceanographic Tables. v.2. Wormley, National Institute of Oceanography of Great Britain \& UNESCO. 